\title{
TTR
}

Traduction, terminologie, re?daction

\section{L'hologramme du poème et le prisme du poétique. Paysages. Mondes possibles. Langage de la pensée}

\section{Pierre Laurette}

Volume 12, numéro 2, 2e semestre 1999

Poésie, cognition, traduction II — Autour d'un poème de W. H. Auden Poetry, Cognition, Translation II — On a Poem by W. H. Auden

URI : https://id.erudit.org/iderudit/037369ar

DOI : https://doi.org/10.7202/037369ar

Aller au sommaire du numéro

Éditeur(s)

Association canadienne de traductologie

ISSN

0835-8443 (imprimé)

1708-2188 (numérique)

Découvrir la revue

Citer cet article

Laurette, P. (1999). L'hologramme du poème et le prisme du poétique. Paysages. Mondes possibles. Langage de la pensée. TTR, 12(2), 13-37. https://doi.org/10.7202/037369ar
Résumé de l'article

L'hologramme du poème et le prisme du poétique. Paysages. Mondes possibles. Langage de la pensée - Une relecture de la rhétorique-poétique est proposée à la lumière des développements récents de la théorie littéraire et de certains aspects des sciences cognitives. La poésie est abordée plus précisément sous le rapport des mondes possibles ainsi que de la traduction-transduction, théorie qui réintègre la sémantique intensionnelle et extensionnelle dans l'analyse littéraire. Toutefois, la poésie moderne ne se laisse pas réduire à cette analyse philosophico-logique. La complexité du langage poétique oblige à envisager aussi la poésie du point de vue de la pensée et du langage (le mentalais) axé non seulement sur le cortical et le cérébral mais également sur l'intuition et les sensations (le limbique).
Tous droits réservés (C TTR: traduction, terminologie, rédaction — Les auteurs, 1999
Ce document est protégé par la loi sur le droit d'auteur. L'utilisation des services d'Érudit (y compris la reproduction) est assujettie à sa politique d'utilisation que vous pouvez consulter en ligne.

https://apropos.erudit.org/fr/usagers/politique-dutilisation/ 


\title{
L'hologramme du poème et le prisme du poétique. Paysages. Mondes possibles. Langage de la pensée
}

\author{
Pierre Laurette
}

\author{
La poétique libérée c'est du bidon \\ Poète prends ton vers et fous-lui une trempe \\ Mets-lui les fers aux pieds et la rime au balcon \\ Et ta muse sera sapée comme une vamp \\ Léo Ferré, « art poétique »
}

L'holographie est l'art de restituer en relief une image en utilisant les interférences de deux faisceaux laser engendrant un rayonnement spatialement et temporellement cohérent. Métaphoriquement, l'un des faisceaux provient de la lecture; l'autre est réfléchi par l'objet, ici le poème. L'essentiel du propos relève cependant du concept holisme selon lequel le tout est quelque chose de plus que la somme de ses parties. D'où les métaphores de poème-hologramme et de poème-cryptogramme.

Le prisme possède la propriété de décomposer, de dévier, de diffracter les radiations lumineuses. Newton découvrit que la lumière blanche est constituée de rayons différemment réfrangibles. Il en va de même des poétiques diverses qui, tel un prisme, projettent un spectre profus de connaissances, de savoirs, de sens, d'intuitions, de suggestions et d'impressions esthétiques. On sait que les ondes incidentes du poème et les ondes réfléchies de la poétique produisent des interférences multiples dont je veux explorer ici quelques franges.

La poesis, l'ars poesis ${ }^{1}$ et l'ars poetica révèlent les aspects les plus complexes de la création humaine. Un long regard rétrospectif sur les arts précités montre à quel point ceux-ci se sont infléchis et leurs lignes se sont brisées au cours du temps. Le terme de poétique, dont le champ

\footnotetext{
${ }^{1}$ L'art d'écrire la poésie, conseil aux jeunes poètes (ex. Rilke, " Lettres à un jeune poète $")$, préfaces, cours didactiques et pragmatiques dispensés par toute personne, compétente ou incompétente, etc.
} 
d'analyse s'est élargi considérablement, a relayé et transformé l'expression arts poétiques. Le descriptif des artéfacts divers, des figures, des tropes, des styles demeure un domaine important dans la recherche en poétique bien que les concepts utilisés aient connu des glissements de sens et que de nombreux néologismes aient été crés pour pouvoir rendre compte de problématiques nouvelles. L'aspect déontique (le il faut de la poétique aristotélicienne et néo-aristotélicienne) a perdu bien de son éclat et a même en grande partie disparu, du moins dans ses manifestations normatives, prescriptives, didactiques et pédagogiques. Le questionnement d'ordre ontologique, philosophique, politique, éthique et cognitif a par contre connu un développement important dans les cinquante dernières années; tout ceci nous oblige à revisiter la poesis et la poétique, l'aventure du sens et les nouvelles perspectives qui se sont depuis ouvertes, en particulier celles présentées par les différents champs du savoir dans les sciences humaines et les sciences cognitives. Quant aux axiologies multiples de l'esthétique, de la littérarité, de la poéticité, du beau, du merveilleux, du sublime, etc., elles demeurent assez énigmatiques et forment de véritables lignes asymptotes vers la courbe insaisissable du poème; elles créent néanmoins un appel d'air et un désir irrépressible de connaissance. On constate de plus l'importance qu'ont pris certains concepts tels que mondes possibles, rythme, écriture, transduction, corps, féminin, sexe, entre autres, l'importance encore des néologismes dans la poétique et la critique dite postmoderne pour décrire, analyser, expliquer, évaluer et interpréter le fait littéraire. La poétique est devenue de nos jours un vaste champ de recherches interdisciplinaires.

Les grands textes littéraires en prose du $\mathrm{XX}^{\mathbf{e}}$ siècle, ceux de Joyce, Kafka, Proust, Hemingway, et naturellement de bien d'autres, sont des ceuvres fortement énigmatiques qui présentent aux lecteurs et aux poéticiens des difficultés de lecture, de compréhension, d'interprétation, de construction, de reconstruction et a fortiori de traduction, de translittération et de transduction..., difficultés créées souvent de manière délibérée, parfois insconsciente, de la part des écrivains, par le biais de stratégies, de dispositifs d'écriture visant ou induisant à éluder toute lecture et interprétation rapide, réductionniste ou définitive. La croisée et l'aventure des sens se nouent et se dénouent dans le texte et sa création, dans la lecture du texte, sa transduction et recréation, dans la contextualisation culturelle et historique et dans le jeu d'innombrables modulations diverses, d'ordre esthétique, ontologique, éthique, logique et axiologique.

La poésie contemporaine - que l'on songe à tel poème de Celan, Char, Montale, Ezra Pound, Joyce, Mallarmé, Mandelstam, Yeats ou de bien d'autres encore, que l'on songe encore à tel poème forgé par les lettristes, les automatistes ou les glossolales pour n'en nommer que quelques-uns - présente des difficultés de lecture, de construction, de reconstruction et d'interprétation tout aussi remarquables par leur énigmaticité que celles du roman, mais elles se situent à un degré quelque peu différent. 
Le champ d'objets ici choisi est le poème court dans la littérature contemporaine. Non pas tant le poème court et bien défini des poétiques anciennes où à l'oratio et aux grands genres du théâtre et du roman on oppose l'idylle, l'élégie, l'épigramme, le rondeau, le madrigal, etc., mais plutôt le poème court, qui dès le $\mathrm{XIX}^{\mathrm{e}}$ siècle se révèle métaphoriquement comme condensateur; au sens physique du terme ou comme sublimation au sens chimique. L'énigmaticité de leur texture tient sans doute au caractère indéfini, ouvert, intensionnel, très peu référentiel, mais aussi à la nature souvent indéfinissable du genre. On constate en effet dans les textes contemporains un certain nombre de tissages et de métissages : a) tissage de faits fabuleux, merveilleux, légendaires et réels ouvrant la voie à des mondes réels, possibles et virtuels et à leur compossibilité; b) métissage générique où s'exerce la générativité de la composition créatrice et où l'éclatement est sans fin puisque les formes les plus extrêmes peuvent naître dans le jeu et le réarrangement : poème-collage, poème calligraphié, poème sans mots, poème syllabique, poème-lettrie, poème-graffito, poème-icône etc.; éclatement encore du support et du médium audio-visuel, et plastique dans le champ scripturaire; c) tissages et éclatements linguistiques que l'on observe par exemple dans les littératures caribéennes où peuvent fusionner les fragments de langue véhiculaire, vernaculaire ou différents sociolectes manifestant une revendication du créateur au droit à la différence; d) tissages et éclatements géolittéraires, géoculturels et géopolitiques dans une dynamique culturelle se caractérisant par un incessant mouvement d'assimilation et de dissimilation culturelles (Laurette et Ruprecht, 1995).

Un bref spicilège donnera un aperçu de cette poésie comme forme expressive la plus complexe de la création artistique par la tension existant entre complétude et incomplétude de forme et de sens, entre caractère holistique et fragmentaire :

Le loriot entra dans la capitale de l'aube.

L'épée de son chant ferma le lit triste.

Tout à jamais prit fin.

(Rene Char)

Dans la bouche de l'hirondelle un orage

S'informe, un jardin se construit

(René Char)

"Mattina »

M'illumino d'immenso

(Ungaretti)

"Eterno "

Tra un fiore colto e l'altro donato

l'inesprimibile nulla

(Ungaretti) 
Un prêtre passe au grand galop

(Cocteau)

Saché-je d'où provient, Sirènes, votre ennui

Quand vous vous lamentez, au large, dans la nuit?

Mer, je suis comme toi, plein de voix machinées

Et mes vaisseaux chantants se nomment les années.

(Apollinaire)

"Un poème "

Il est entré

Il s'est assis

II ne regarde pas le pyrogène à cheveux rouges

L'allumette flambe

Il est parti

(Apollinaire)

Je la connus Ah merdemore

Ensoleillée le dix-neuf août

Je la crus Béatrice ou Laure

Amour et gloire J'étais fou

Lauriers ô touffes d'ellébore

(Apollinaire)

Mon récit sera la branche noire

qui fait un coude dans le ciel

(André du Bouchet)

"Agua Dormidor"

Quiero saltar al agua para caer al cielo

(Pablo Neruda)

"Mi alma "

Mi alma es un carrousel vacio en el crepusculo

(Pablo Neruda)

Ihr hohen Pappeln - Menschen dieser Erde!

Ihr schwarzen Teiche Glücks - ihr spiegelt sie zu Tode!

Ich sah dich, Schwester, stehn in diesem Glanze.

(Paul Celan)

Nous avons envoyé contre eux un seul Cri

et ils devinrent semblables

à l'herbe desséchée d'un enclos.

Le Coran (sourate LIV, verset 31)

La chargougne s'étalait tout autour de la ville, boursouflée de magrassine. Entassement de grumoulises barlinguées de crabouches pestilentielles. Foumarie patafoumeuses de golinces (FrankÉtienne)

Le champ théorique de la poétique offre une mine tout autant fabuleuse que celle du champ d'objets par l'élargissement des savoirs et la 
complexification qui s'y rattache. Les connaissances sur et à partir de l'œuvre littéraire ne cessent de s'enrichir et de dérouter ceux qui visent à atteindre une intelligibilité globale de la création littéraire et artistique. La diversité des poétiques découle certes au premier chef du renouvellement constant des œuvres littéraires, de leurs formes, mais elle provient aussi de la refonte des domaines du savoir et de la recombinaison constructive entre disciplines, voisines ou éloignées.

Il y a tout d'abord les théories textuelles et littéraires qui sont de l'ordre de la description, de l'interprétation et de l'évaluation. Les poétiques et les rhétoriques aristotéliciennes et néo-aristotéliciennes, dont les domaines se recoupent en partie dans l'inventio, la dispositio et l'elocutio (Lausberg, 1960, p. 42), constituent sans doute la trame conceptuelle et la grille d'analyse la plus détaillée. ${ }^{2}$

La poétique, comme champ spécifique, est bien le lieu de diverses recherches entretenant de nombreux liens interdisciplinaires et polysegmentaires avec d'autres champs du savoir (Laurette, 1993) ${ }^{3}$ Dans les poétiques que l'on peut considérer comme science de la littérature, comme champ de recherches cognitives, interviennent différents savoirs. Les uns sont traditionnellement assez spécifiques tels que la poétiquerhétorique, la sémiotique, la linguistique, voire la stylistique, domaines classiques de la poétique. Les autres, de nature exogène venant de l'ensemble des sciences humaines, marquent la fin de la clôture du texte. L'analyse, l'interprétation, l'évaluation ont recours à la philosophie, la sociologie, l'histoire, l'anthropologie, la psychanalyse et à l'ensemble des champs disciplinaires en sciences humaines, et même à certains domaines des sciences exactes et des sciences informatiques et cognitivistes récentes telles l'intelligence artificielle, la psychologie cognitive, les représentations mentales, les combinatoires, les connectionnismes, les théories computationnelles. Ainsi s'ouvrent de nouvelles perspectives et recherches multiples touchant de manière sans doute encore balbutiante au domaine de la littérature et de l'art.

Les recherches sur le rythme sont à ce propos topiques et exemplaires. Elles traduisent l'ouverture aux différents savoirs disciplinaires. Ce concept est de nature microcosmique quand il relève de son objet, le poème, et il est, dans les poétiques, de nature macrocosmique : relations au corps, au monde, au surnaturel, à la tradition, à la culture et à la mémoire. De nombreux champs du savoir s'y greffent, tels que la psychanalyse, l'anthropologie, la psychophonétique, la

${ }^{2}$ Les dictionnaires de poétique et de rhétorique offrent un éventail de termes plus ou moins riche. Une étude comparée des dictionnaires de poétique et de rhétorique dans différentes cultures et littératures permettrait de relancer le débat sur la validité de la poétique-rhétorique.

3 Voir en particulier le chapitre 2, "Littérature, pluridisciplinarité et technologie ». 
sémiotique, la phénoménologie, la linguistique et la poétique générativistes ou les sciences cognitives (dans la perception et la production du rythme). ${ }^{4}$

Il existe ainsi un véritable champ de tensions pluridisciplinaires. La théorie littéraire, conçue dans son sens le plus large, se situe dans la mouvance de ce vaste ensemble polysegmentaire que constituent les sciences de l'homme et de la société et aussi de nouvelles sciences en voie d'émergence telles les sciences cognitives (domaine fortement agrégatif lui-même) qui entre autres choses s'occupent de représentations, de phénomènes mentaux, du langage de l'esprit (mentalais) et d'entités ontologiques fort diverses dépassant le champ d'une science particulière comme la sémiotique, la rhétorique, la linguistique, etc. Est-il possible de rester confiné plus longtemps dans le découpage disciplinaire traditionnel des institutions universitaires? Les frontières disciplinaires existent encore, certes, mais au détriment souvent du réseau, des connections réelles, possibles, virtuelles et des échanges d'informations qui sans cesse renouvellent questionnements, savoirs et connaissances. Comme le souligne Dan Sperber (1992, p. 399) : « La réalité de la recherche est bien plutôt celle d'un réseau où les échanges intellectuels dessinent et redessinent des aires d'activité intense, petites ou grandes, incomplètement séparées les unes des autres par des zones de moindre communication, d'ignorance mutuelle ou, parfois, d'échanges hostiles ". On ne peut qu'être frappé par le déploiement des concepts et des théories engendrant une dynamique de la différenciation, de la dissimilation sans fin dans le champ de la critique littéraire, celle-ci devenant une diaphore générale (Caravetta, 1991). Diaphore: une figure où l'on répète un mot avec une nouvelle nuance de signification et d'interprétation. Ici je conçois la diaphore comme paradigme de concepts où la générativité est quasi infinie, car elle repose non seulement sur l'utilisation assez systématique des ressources morphologiques d'une langue naturelle donnée, mais encore sur des hypothèses profuses d'interprétations, d'herméneutiques d'inspirations diverses. Le déconstructionnisme de la critique dite postmoderne laisse d'innombrables traces dont j'ai analysé le spectre et les tendances dissimilatrices dans le champ de la critique postmoderne (Laurette, 1996). Les concepts préfixés en de, dia, dis, meta, post, inter, trans, para, etc. sont légions. Ces catégories préfixales de langue sont médiatrices de catégories de pensée par subduction, par appropriation sublogique; elles sont de nature diaphorique et ont une fonction heuristique.

La poésie, ce mystère dans les lettres, "arcane étrange » qu'évoque Mallarmé $a$, presque de tout temps, tenu une position hors norme par rapport à la langue : « Le vers qui de plusieurs vocables refait un mot total, neuf, étranger à la langue et comme incantatoire, achève cet

4 Pour le seul domaine français, voir entre autres les travaux de Kristeva, Fonagy, Jousse, Zilberberg, Roubaud, Meschonnic. 
isolement de la parole [...] » (Mallarmé, 1945, p. 368). Platon n'estimait guère la fantasmagoria du poète, qui ne trouvait pas sa place dans sa République. Cette situation étrange de la poésie est ainsi posée par Jean Paulhan :

Voici le lieu le plus commun du vague et de la contradiction. On commence, à l'ordinaire, par admettre qu'il est, au ccur de la poésie, un mystère proprement indicible. Cependant, l'on traite ensuite du détail de cette poésie, par lois et par règles [...]. Or si le mystère est essentiel à la poésie, [...] chaque trait poétique, de la rime à l'exercice spirituel devrait au contraire porter la marque de ce mystère, et le traduire en quelque façon. (1966, p. 241)

Cette élévation, par degrés, de la poésie par rapport au langage peut être ainsi résumée : le langage est conçu comme "métasystème culturel » (Hagège, 1994, p. 18), la littérature comme " système modelant secondaire " par les sémioticiens de Tartu et enfin, dans un sens plus transcendant, la poésie est ressentie comme " puissance seconde 》 (zweite Potenz par Novalis, 1946, p. 93). C'est aussi la position ontologicopoétique de Heidegger $(1982,1984)$ pour qui la langue du poète est aletheia; la vérité du mot, l'ivresse et l'action du mot dans la poésie débouche sur l'ouverture, l'ouvert (das Offene). Le poète est alors un demi-dieu (Halbgott). Dieu, demi-dieu, chaman, barde, griot, glossolale, schizophrène, logophile ou fou de la littérature !... autant de masques, de personae par où s'exprime la Pythie et la parole poétique sur le mode de la furor, de l'illumination et de la révélation !

La poésie ne pourrait-elle être alors déclinée que sur le seul mode négatif, sur le modèle de la théologie négative où toute assertion est ressentie comme borne, comme limite? La litanie serait longue... Dans les pages qui suivent je me propose d'examiner certaines franges de la poétique : 1) le paysage comme concept utilisé en poétique et dans les sciences humaines; 2) la langue de la pensée ou mentalais (dans les sciences cognitives); 3 ) le poème-cryptogramme et les mondes possibles; 4) la poésie comme transduction.

\section{Paysages}

« Paysage "

Paysages paisibles ou désolés.

Paysages de la route de la vie plutôt que de la surface de la Terre.

Paysages du Temps qui coule lentement, presque immobile et parfois comme en arrière.

Paysage des lambeaux, des nerfs lacérés, des «saudades».

Paysages pour couvrir les plaies, l'acier, l'éclat, le mal, l'époque, la corde au cou, la mobilisation.

Paysages pour abolir les cris.

Paysages comme on se tire un drap sur la tête.

(H. Michaux) 
Pour éclairer certains traits de l'écriture énigmatique de la poésie j'avancerai provisoirement comme prétexte heuristique et ludique un concept à valeur, à première vue, analogique ou métaphorique, à savoir celui de paysage dont on peut tenter de décliner certains sens et perspectives. Le concept de paysage est par excellence holistique, mais il peut s'entendre de plusieurs façons. Le poème peut être vu comme paysage en soi, comme monde doté de traits réels, irréels, surréels, allégoriques, etc. Ce poème peut être envisagé comme texte situé dans un paysage culturel et contextuel donné, débordant la clôture textuelle. Il peut encore se concevoir comme paysage s'ouvrant sur un paysage personnel, la pensée et le langage de la pensée d'un lecteur qui le reconstruit. Le mot paysage peut enfin être utilisé comme méta-concept ayant une fonction descriptive, analytique, holistique et heuristique. On peut ainsi noter que ce concept a déjà été utilisé à titre métalinguistique par des chercheurs en lettres et sciences humaines : "Landschaft als interdisziplinäres Forschungsproblem » (Le paysage comme problématique dans la recherche interdisciplinaire) ${ }^{5}$. Dans cette étude le concept est utilisé dans le cadre d'une réflexion interdisciplinaire touchant aux domaines de l'esthétique, de l'art, de la littérature, de la géographie, de l'histoire, du droit et de la politique. Thomas Pavel a utilisé les expressions paysage fictionnel et paysage ontologique pour illustrer de manière holistique les propriétés globales qu'entretiennent l'univers de la fiction et le monde de l'ontologie globale d'une époque (1988, pp. 175176).

Le concept d'écologie du paysage - on pourrait créer le calque écologie du paysage littéraire-a été utilisé dans les sciences écologiques pour tenter de comprendre les structures complexes du paysage considéré dans la totalité de sa biodiversité : dans l'atmosphère, l'hydrosphère, la lithosphère, l'éconosphère, tout ceci sous la lumière solaire, et dans l'organisation détaillée du paysage lui-même (réseau de haies, de sentes, de voies, de rivières, distribution d'îlots, de terrains vagues, de paysage organisé, de bois, organisation des différents étages d'une forêt, forme et composition des lisières ou de forêts riveraines, de fleuves, d'alternance de cultures, de friches, de boisements, organisation de l'habitat, de l'éconosphère, etc. Tous ces traits du paysage saisi dans sa biodiversité sont corollaires de la diversité génétique elle-même).

La littérature est considérée comme paysage que l'on ne peut englober d'un seul regard. L'holistique, dans sa prétention à l'exhaustivité, est du domaine du divin ou de la métaphysique. Le concept de paysage ontologique est avant tout une métaphore dont je veux dire deux mots. «Un paysage, écrit Fontenelle, dont on aura vu toutes les parties l'une après l'autre, n'a pourtant point été vu, il faut qu'il le soit

${ }^{5}$ Titre d'un colloque (Münster, 7-8 novembre 1975) réunissant des spécialistes de différents domaines tels que la littérature, la géographie physique et humaine, la linguistique (étymologie du mot paysage), l'histoire, l'histoire de l'art, les sciences politiques et sociales, etc. 
d'un lieu assez élevé, où tous les objets auparavant dispersés se rassemblent sous un seul coup d'oeil ». Madame de Sévigné parlant d'un de ses voyages écrit: " Nous voyons deux mille objets différents qui passent incessamment devant nos yeux comme autant de paysages nouveaux ". La diversité des éléments d'un poème et son unité en tant qu'objet sont comme le simulacre d'un paysage : en peinture, par ce qu'il révèle dans sa totalité et par ce qu'il cache par inachèvement dans ses creux, ses ombres, ses perspectives cachées, par ce qu'il permet ainsi d'imaginer, de rêver, de recréer. Analogiquement, pour le caractère holistique, on peut penser aux toiles de la peinture flamande et francoflamande du XVe siècle chez Van Der Weyden, Marmion, Bosch; pour le caractère fragmentaire, on pourrait évoquer Odilon Redon et de nombreux peintres contemporains.

Le statut ontologique de la poésie traduit ce besoin fondamental de comprendre l'être, l'existence. Chez de nombreux poètes et philosophes on ne peut parler de la parole sans poser le problème de la transcendance de l'être et, selon Cicéron, de la magistrature de la parole. Parménide, Héraclite, Empédocle illustrent particulièrement ce questionnement. Heidegger, à travers l'œuvre d'Hölderlin, trouve dans la poésie la révélation miraculeuse de l'être, son dévoilement, l'intuition absolue de l'être. Hésiode, Héraclite et René Char révèlent dans leurs œuvres la cohérence absolue de l'être et de l'apparence, de l'un et du multiple qui coïncident.

L'ontologie, comme partie importante de la philosophie et de toute réflexion sur l'art, est quant à elle, inhérente à la poésie et aussi en quelque sorte en surplomb par rapport aux modalités de vérité, de morale, de connaissance et de valeurs axiologiques diverses par l'énigme et la problématique qu'elle pose : être et apparence, amour et haine, tous les lieux pathétiques, la mesure, l'hybris, la plénitude de l'un, les contradictions du multiple, le beau, le vrai, le délire, le bon, la vertu, toutes les entités du monde sacré, profane, animal, végétal, etc. Le mode ontologique de la littérature, son être, son existence dans le langage, dans l'œuvre littéraire, l'ontologie des êtres, des mondes fictionnels, constituent paradoxalement ce qui est et ce qui n'est pas. Cette problématique est centrale dans les recherches de la poétique. Je renvoie entre autres aux travaux de Lubomir Doležel et de Thomas Pavel consacrés essentiellement aux romans et aux récits, aux mondes possibles et aux mondes fictionnels.

Le paysage ontologique de tel écrivain ou de telle œuvre est plus ou moins flottant, plus ou moins dessiné. Il se réalise toujours d'une manière composée, et sa netteté est d'autant plus grande que l'ontologie de l'écrivain et de l'époque est forte et relativement unitaire. Les modalités précitées sont ainsi, chez un auteur comme Cicéron, d'une assez grande clarté : épistémique - Cicéron est un des fondateurs de la conception moderne de la culture; il réclame une culture universelle, quasi encyclopédique pour la magistrature de la parole; déontique - il croit à la 
splendor honestatis, à la splendor virtutis; il est apte à la compassion, à la misericordia, à la caritas héroïque, supérieure à l'amour; aléthique - la recherche constante de la vérité; on peut croire le témoin qui se fait tuer. D'autres exemples de netteté du paysage ontologique peuvent être donnés ainsi dans nombre d'œuvres néo-platoniciennes où se combinent harmonieusement le beau, la vérité et l'amour, le beau qui selon Platon est la splendeur du vrai. Il est, je crois, plus difficile de dresser le paysage ontologique de telle ou telle cuvre d'écrivains modernes. Car l'hétérogénéité ontologique est un des traits les plus marquants de toute une grande partie de la littérature contemporaine, à l'exception peut-être de certaines littératures d'édification morale unilatérale, du roman sentimental dit « Arlequin » et des feuilletons globalisés.

Le concept d'inscape du poète G. M. Hopkins est un autre avatar du concept de paysage: de nature esthétique, métaphysique et profondément individuelle par sa forme intensionnelle. L'esprit découvre à partir des apparences le dessein intérieur et caché des choses, et sa marque intérieure, l'instress, trouve le rythme et l'ordre caché. Il s'agit de voir au-delà des images, des mots. Le rythme est le moyen de pénétrer et de traduire la réalité du monde :

We must judge by the ear, not by reading, and the eye. [...] We may now say of rhythm, i.e. verse that it is the reading of speech into soundwords, sound-clauses, and sound-sentences of uniform commensurable length and accentuations. [...] It almost seems as if the rhythm were disappearing and repetition of figure given only by the alliteration[...] This beat-rhythm allows of development as much as time-rhythm wherever the ear or mind is true enough to take the essential principle of it; that beat is measured by stress or strength, not number, so that one strong may be equal not only to two weak but to less or more. (Hopkins, 1959 , p. 235)

On peut noter qu'en anglais le mot landscape, par sa morphologie, se prête à des variations linguistiques et sémantiques interressantes. Dans son ouvrage Landscape of the Mind, J. Douglas Portevus (1990) intitule ses chapitres Smellscape, Soundscape, Bodyscape, Inscape, Homescape, Childscape, Deathscape, Otherscape.

\section{Le langage de la pensée ou mentalais}

Les sciences cognitives constituent un vaste champ de recherches non unifié qui se distingue avant tout par la modularité des champs du savoir et leurs connexions multiples. Flles constituent une véritable "galaxie " réunissant des chercheurs venant d'horizons aussi divers que la linguistique, l'anthropologie, la philosophie, la logique, l'intelligence artificielle, l'informatique, la neurobiologie, la neuropsychologie, etc. Le champ d'objets des sciences cognitives embrasse la cognition au sens large et les problèmes qui en relèvent : langage de la pensée, acquisition du langage, images mentales, apprentissage, perception, mémoire, intelligence, représentations symboliques, raisonnement, conscience, 
processus d'attention, créativité, processus de l'action, émotions, etc.

La création artistique et les poétiques sont-elles concernées par les hypothèses et la problématique de cette science pluridisciplinaire encore naissante? Jusqu'à un certain point, oui, et en particulier par les problèmes suivants :

a) Pense-t-on avec des mots? C'est là évidemment un vieux problème philosophique auquel des éléments de réponse ont été apportés.

b) Quels sont les types et modes de relation existant entre esprit et cerveau? Mind/brain? Le sens anglais de brain est moins large que le concept d'esprit.

c) Quels sont les états internes ou mentaux de la représentation? Quels sont les processus de création en dehors des mots? Quels sont les processus de construction dans la lecture et la transduction?

d) Comment se réalisent les transductions de ces états mentaux avec ce qui est externe?

e) Comment fonctionne le langage de la pensée dans la poïesis? La poïesis se jouant à la fois en deçà des mots, par symboles et représentations, par les mots de la langue et au-delà de ces mêmes mots.

Ce sont certes des problèmes qui sont loin d'avoir trouvé une solution, mais qui permettent cependant de s'interroger sur la validité de certains traits ou sur certains aspects de la poétique-rhétorique, sur la théorie des signes, sur la portée réelle de la sociologie littéraire, sur la transduction, les modulations, sur la générativité dans le langage de la pensée.

Le mentalais selon S. Pinker est « un langage hypothétique de la pensée ou représentation de concepts ou de propositions dans le cerveau dans lequel se situent (are couched) les idées, incluant le sens des mots " (1994, p. 478). Selon le même auteur, le cerveau humain utiliserait au moins quatre formats de représentation: l'image visuelle, la représentation phonologique, la représentation grammaticale et le mentalais (mentalese) qui serait le médium où se situe, est saisi le contenu ou l'essentiel (l'esprit ou gist) (1997, p. 19). Pinker compare encore le mentalais à quelque lingua franca où se réaliserait "le trafic de l'information à travers les modules mentaux $॥$; cette circulation de l'information serait empiriquement observée dans le cerveau, ce connectoplasme dont on ne sait encore que peu de choses.

Ce qui frappe le plus, c'est de constater la complexification quantitative et qualitative de ces processus dans le langage des mots, des phrases et enfin de la pensée. La création de mots et de néologismes est quasiment illimitée, celle des phrases encore plus grande par 
combinatoire et générativité. Quant au mentalais, sa combinatoire est d'une vastitude inimaginable: "The combinatories of mentalese, and other representations composed of parts, explain the inexhaustible repertoire of human thoughts and action. ") (1997, p. 17). Les générativistes pensent que le cerveau est doté d'une grammaire universelle et qu'ainsi la combinatoire des mots et des pensées est sans fin. On peut donner une illustration de ce pouvoir génératif à créer des mots par dérivation, par analogie, par jeu... les mots manquent, ils se créent :

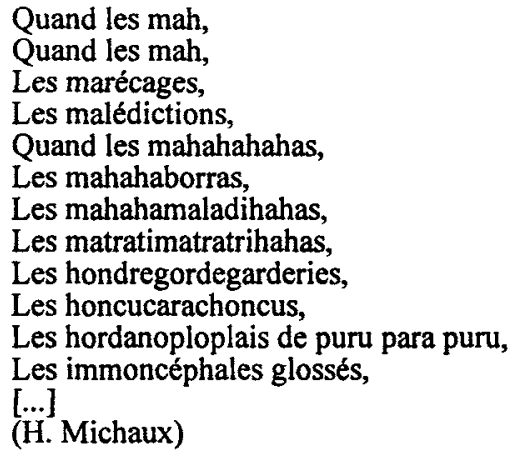

Il l'emparouille et l'endosque contre terre;

Il le rague et le roupète jusqu'à son drâle;

Il le pratèle et le libucque et lui barufle les ouillais;

Il le tocarde et le marmine,

Le manage rape à ri à ra.

Enfin il l'écorcobalise.

(H. Michaux)

Le signe n'est pas la conséquence des choses "signa sunt consequentia - rerum " - conception néoplatonicienne; le signe et la syntaxe ne déterminent pas les choses comme dans l'hypothèse de SapirWhorf, conception déterministe linguistique. Le signe se greffe en deçà ou au-delà du mot lui-même, par représentation symbolique dans le mentalais. Les lieux et les liens entre les mots, les symboles et le sens essentiel, entre le cortex et le système limbique sont encore en grande partie terra incognita dans la mappemonde du cerveau. John Searle (1995) avance l'idée, d'un point de vue épistémologique, que l'utilisation de la troisième personne, le $i l$, d'allure plus objective, " ne doit pas nous rendre aveugle au fait que l'ontologie réelle des états mentaux est une ontologie à la première personne $"$.

La mémoire de la pensée, en particulier, est la manifestation la plus intensionnelle et singulière de l'individu. Elle ne peut, dans la construction artistique ou dans la reconstruction par la lecture, faire appel à quelqu'encyclopédie comme le conçoit Umberto Eco, ni se satisfaire 
d'une encyclopédie fictionnelle telle que le conçoit Lubomir Doležel (1998). L'encyclopédie du mentalais est infinie dans la mesure où elle opère avec les mots et hors des mots de la langue, avec les représentations symboliques internes.

De nombreuses notions peuvent se concevoir en dehors des mots mêmes : l'espace, le temps, le nombre, la fréquence, la causalité, les catégories, la pensée visuelle, la représentation symbolique interne et bien d'autres entités comme le rythme, les sons, les esthésies et synésthésies, les caractères prosodiques, les évaluations axiologiques diverses peuvent être de nature supralangagière. La création dans le domaine des lettres comme dans les autres domaines scientifiques est exécutée parfois de façon supralangagière. On connaît les cas de nombreux inventeurs et créateurs tels S. T. Coleridge, A. Einstein, M. Faraday, P. Valéry et de bien d'autres qui ont conçu certaines de leurs œuvres grâce à des images mentales plus ou moins claires et non par des mots. Paul Valéry, dans Mémoires d'un poème, évoque cette création antérieure au langage :

Tel poème a commencé en moi par la simple indication d'un rythme qui s'est peu à peu donné un sens. Cette production, qui procédait, en quelque sorte, de la "forme" vers le "fond", et finissait par exciter le travail le plus conscient à partir d'une structure vide, s'apparentait, sans doute, à la préoccupation, qui m'avait exercé, pendant quelques années, de rechercher les conditions générales de toute pensée, quel que soit son contenu.

Ou encore : «[...] l'état naissant des poèmes peut être très divers : tantôt un certain sujet, tantôt un groupe de mots, tantôt un simple rythme, tantôt (même) un schéma de forme prosodique, peuvent servir de germes et se développer en pièce organisée ». Dans l'Idée fixe, Valéry forge le néologisme implexe ou encore le concept de nébuleuse mentale, qui par plusieurs traits rappelle le mentalais. L'implexe chez Valéry est un ensemble « d'incidents et d'apparences incohérentes : sensations, images de tous genres, impulsions, mots isolés, fragments de phrase... ". Celui-ci évalue "l'incalculable réseau » que constitue le moindre texte littéraire. Ainsi, pour Valéry, l'art littéraire " dérivé du langage " est " de tous les arts, celui qui engage et utilise le plus grand nombre de parties indépendantes (son, sens, formes syntaxiques, concepts, images ...) ). La recherche valéryenne sur ce qu'il nomme implexe ne se fait cependant que de manière phénoménologique en dehors des sciences linguistiques, neurologiques et psychoneurologiques. La pauvreté du vocabulaire en psychologie est frappante selon Valéry:

Il ne faut jamais laisser échapper une occasion de souligner la grande misère du vocabulaire "psychologique". Quoique un peu moins pauvre que la nôtre en termes de cet ordre, la langue anglaise ne permet pas mieux de distinguer ce que nous voudrions discriminer, ce que nous essayons de discerner par les mots : psychique, mental, intellectuel...

Les connaissances en sciences cognitives se précisant de plus en 
plus sur le langage de la pensée - langage incommensurable par sa combinatoire et sa générativité, ce que reflète le poème -, ne remettentelles pas en question la validité de certains aspects de la rhétorique? Jean Paulhan remarquait cette incapacité de la rhétorique à analyser certaines expressions littéraires et certains fragments textuels: «On citerait aisément mille autres exemples, où l'expression dans son jeu semble échapper aux classes et catégories rhétoriciennes, à la fois par le haut et par le bas - par l'extrềme conscience comme par l'extrême inconscience » $(1966$, p. 12).

Par le haut, ici l'on peut interpréter dans un sens plus large en spécifiant le domaine des modalités ontologiques, déontiques, épistémiques, esthétiques, aléthiques, etc., et par le bas, en songeant à l'articulation: langage, mots, mentalais, représentation symbolique, système cortical et système limbique :

"Rubililieuse"

Rubililieuse et sans dormantes,

Vint cent Elles, Elle, Elle,

Rubililieuse ma bargerie,

Nous contre, noue, noue,

Ru vaignoire ma bargerie.

(H. Michaux)

\section{Poème-cryptogramme et mondes possibles}

La problématique des mondes possibles est fort ancienne du moins sous la forme de thèse philosophique, de pluralité des mondes : " an mundi multi sint ». Les mondes mythiques, les légendes, les grands textes religieux, les littératures mettent en scène des mondes merveilleux, fantastiques, surréels, aliénés, paranormaux et de nombreux mondes hybrides, métissés avec le monde réel, actuel. Dans ses Entretiens sur la pluralité des mondes habités, Fontenelle se divertit à peupler « d'Habitans une infinité de Mondes " et précise :

Je n'ai rien voulu imaginer sur les Habitans des Mondes, qui fût entièrement impossible et chimérique. J'ai tâché de dire tout ce qu'on en pouvait penser raisonnablement, et les visions mêmes que j'ai ajoutées à cela, ont quelque fondement réel. Le vrai et le faux sont mêlés ici, mais ils y sont toujours aisés à distinguer . (1991, p. 12)

Dans le monde fictionnel il est difficile de distinguer valeur de vérité et imagination secrètement entrelacées. Selon Leibniz, notre monde est entouré d'une infinité d'autres mondes possibles, mais ce qui nous intéresse ici, chez Leibniz, c'est la poïesis créatrice, poïesis par la combinatoire exposée dans sa Dissertatio Arte combinatoria et par sa logique " plus sublime ", logique de la mathématique universelle, logique de l'invention non seulement des faits déjà connus mais surtout des vérités nouvelles. L'art combinatoire est illustré chez Leibniz par l'artéfact des 
cadenas à secret. Le poème moderne est analogiquement un cadenas à secret. La théorie littéraire du monde fictionnel a réutilisé de manière féconde la problématique des mondes possibles. Les travaux entre autres de L. Doležel $(1990,1998)$ et de T. Pavel (1988) ont contribué à créer de nouvelles perspectives théoriques dans le domaine du roman.

La problématique ici envisagée pour analyser le champ des poèmes courts est marquée par quatre concepts majeurs : monde réel ou actuel, monde possible, monde fictionnel, monde virtuel. La conception d'un monde réel ou actuel comme unique source d'inspiration pour la poésie n'est en aucun cas satisfaisante, car elle est incapable de rendre compte de toute la réalité, de la forme et du sens qu'est le poème, ni d'évaluer les aspects autotéliques, autoréférentiels, génératifs, ludiques et intensionnels de l'écriture. La critique de la mimesis est récurrente dans la critique littéraire postmoderne. Mais le monde actuel est une nécessité pour mettre en perspective certains traits du poème et pour évaluer consciemment ou inconsciemment toute forme de distanciation et de différenciation. Le jeu entre sémantique référentielle (Bedeutung chez Frege) et la sémantique intensionnelle, celle qui est donnée par le texte lui-même, par l'intégralité de sa texture (Sinn chez Frege; l'effet de sens, la forme-sens chez Meschonnic) est une dimension importante de la poétique, non seulement dans la construction chez l'écrivain, mais aussi dans la reconstruction chez le lecteur ou le traducteur.

La poétique des mondes possibles est l'expression abstraite de ce qui est pensable; le possible est une catégorie essentielle de la modalité dans la logique classique (possible/impossible). Des hommes à tête de chien peuvent constituer l'idée séminale d'un monde possible pour un roman encore non-réalisé et qu'on pourrait se proposer d'intituler L'île des hommes à tête de chien, même si cette idée séminale est tirée du Livre des merveilles de Jean de Mandeville. Tout cela relève de la problématique des mondes possibles; ce projet ne fait pas encore partie du monde fictionnel, qui est un univers construit de manière plus ou moins contraignante. L'exemple précité montre, d'une part, qu'il n'existe pas de mondes impossibles, d'autre part qu'il faut, dans le monde fictionnel du roman, établir une certaine complétude, une certaine saturation grâce aux personnages, à l'intrigue, aux événements, aux péripéties, au dénouement, etc. Le poème court, par sa concision même, est en quelque sorte insaturé ou poly-insaturé, il peut jouer sur le mode incomplétude/complétude :

Auberge de boues molles pour poissons passant la nuit hors de l'eau.

(H. Michaux)

Souricières de l'âme après extinction du calorifère

blanc méridien des sacrements

Bielle du navire

Radeau

Jolies algues échouées il y en a de toutes couleurs 
Frissons en rentrant le soir

Deux têtes comme les plateaux d'une balance.

(A. Breton, P. Soupault)

Le monde virtuel est caractérisé par une actualisation, un passage de la puissance à l'acte, de la virtualité à la réalité du texte écrit. Il se manifeste dans le processus de transduction, de construction et de reconstruction par le lecteur ou le traducteur, processus évoqué plus loin. De nombreux poèmes ont pour caractéristique dominante le côté hybride et flottant entre ces mondes : réel, possible, virtuel et fictionnel :

Un prêtre passe au grand galop

(Cocteau)

Image, certes, mais le possible côtoie un aspect fictionnel que l'interprétation pourrait virtuellement développer en un monde fantasmatique, idéologique ou autre...

L'expression poème-cryptogramme éclaire certains traits de l'opposition complétude/incomplétude, résolution/irrésolution du sens. Dans son Art d'inventer, Leibniz expose le problème du déchiffrement d'un cryptogramme. Il remarque que si le cryptogramme (je pense ici par analogie au poème court) ne contient pas suffisamment de lettres ou de chiffres pour déterminer la clef, il admet de la sorte une multitude finie ou infinie de clefs et par conséquent d'interprétations différentes plus ou moins probables. Si le cryptogramme a une certaine longueur, il fournira juste les données nécessaires et suffisantes pour déterminer la clé (la solution, l'interprétation). Enfin si le cryptogramme dépasse cette longueur minimum pour trouver la clé, les données seront surabondantes, surnuméraires, plus que suffisantes et elles permettront de déterminer la clef de plusieurs façons différentes. Leibniz en fait la démonstration très simple par la géométrie. Le caractère cryptographique de certains poèmes modernes courts devrait être reconsidéré, en reprenant les idées de Leibniz sur la science des possibles et sur le calcul des probalités, comme probabilité totale (c'est-à-dire alternative d'événements ou addition des probabilités) ou comme probabilité composée beaucoup plus complexe (multiplication des probabilités entre elles). Une autre comparaison de Leibniz peut fournir un éclairage un peu différent. Il s'agit encore une fois du cadenas à secret - le poème comme cadenas à secret. Le cadenas est constitué de plusieurs tambours ou cercles où sont inscrits un grand nombre de chiffres ou de lettres, d'où il découle un très grand nombre de combinaisons possibles. Mais ce cadenas ne possède qu'une seule clé chiffrée de sorte qu'il est hautement improbable qu'on puisse trouver la solution.

Le poème-cryptogramme ou le cadenas à secret joue sur des modalités diverses telles l'ontologie, les valeurs de vérité (aléthiques), les valeurs déontiques et les valeurs axiologiques en un vaste spectre. Le poème existe, se révèle par différents modes, par la voix, 
l'impersonnification où le poète narrateur peut parler comme à travers un masque, une persona, qui s'exprimera comme prophète, griot, barde, illuminé, comme tribun, gourou, schizophrène, révolté, anar, parano, Job ou comme toute autre personne ou symbole par intériorisation énonciative. Dans le domaine de la poésie, les modalités : aléthique (vrai/ faux/plausible), déontique (permis/interdit/obligatoire/toléré), axiologique (valorisation/dévalorisation) qui touchent plusieurs domaines (moral, esthétique, idéologique, politique), se prêtent assez mal à une évaluation de nature logique.

Le jeu de ces modalités, les dichotomies extensionnelles/ intensionnelles, tendent à établir une probabilité composée et complexe quant au sens ou à l'évaluation du sens, comme on peut le voir dans ce poème déjà cité :

\section{Je la connus Ah merdemore Ensoleillée le dix-neuf août Je la crus Béatrice ou Laure Amour et gloire J'étais fou Lauriers ô touffes d'ellébore. (Apollinaire)}

La logique de Leibniz fournit d'ailleurs les concepts qui atténuent et relativisent les modalités sévères du "vrai ou faux " ou de toute autre modalité. À côté des deux quantités aristotéliciennes Universelle et Particulière, Leibniz introduit deux autres quantitésqualités Singulière et Indéfinie. Ce dernier concept est topique, en particulier pour éclairer le fonctionnement des modalités aléthiques (vrai, faux) concernant les mondes réels, fictionnels et possibles dans la réalité et la littérature. La logique humaine est multivalente et à côté de la logique binaire et ternaire, on peut imaginer et réaliser d'autres logiques capables d'exprimer notre pensée, nos axiologies qui sont nuancées, floues, fluctuantes. Ainsi, en logique quinaire, on distingue cing gradations (faux/plus faux que vrai/ni faux, ni vrai/plus vrai que faux/ vrai), en logique endécadaire, onze gradations (faux/quasiment faux/ presque faux/assez faux/plus faux que vrai/ni faux, ni vrai/plus vrai que faux/assez vrai/presque vrai/quasiment vrai/vrai). En fait, les logiques naturelles sont multivalentes et permettent le jeu d'inférences multiples et d'intervalles de confiance, si l'on veut élargir encore l'imprécision dans l'acte de la création, de la lecture, de l'analyse et de la traduction littéraire. Les ensembles flous de Zadeh conviennent mieux au fait littéraire, aux mondes possibles, réels, fictionnels ou virtuels et aux «tonalités flottantes » dont j'aborde plus loin quelques aspects.

\section{Poésie et transduction}

Le préfixe trans est très productif dans le discours philosophique et en théorie littéraire:transfert, transformation, transgression, translation, 
translittération. Dans la critique postmoderne, j'ai relevé les termes suivants : transmétrisation, transmodalisation, transmotivation, transtextualité, transstylisation, transvalorisation, etc. J'ai de plus relevé dans deux corpus différents que, par ordre de fréquence décroissante, les concepts de la critique littéraire postmoderne forgés avec le préfixe trans venaient respectivement au troisième rang (de, dia, di, inter, trans) et au cinquième rang (de, dia, di, post, meta, anti, trans) (Laurette, 1996).

Le traducteur de poésie moderne et énigmatique, quand bien même il s'efforce de garder l'esprit de l'intensionnalité textuelle, se voit parfois contraint de rendre plus clair ce que le texte propose. Il fait œuvre, consciemment ou inconsciemment de métaphraste malgré lui. ${ }^{6}$ Le terme de transduction littéraire utilisé par $\mathrm{L}$. Doležel a pour fonction d'embrasser la sémantique à deux valeurs, référentielle et intensionnelle, dans le cadre de la communication littéraire et de montrer la chaîne illimitée de cette transduction. Il propose un modèle qui illustre clairement ces deux aspects (Doležel, 1998, p. 204). L'auteur 1 écrit le texte 1 et construit le monde fictionnel 1 ; le lecteur 1 lit le texte 1 et reconstruit le monde fictionnel 1; la concaténation se poursuit avec l'auteur 2, le lecteur 2 et le monde fictionnel 2 et ainsi de suite; la deuxième concaténation se réalise, non du texte 1 à 2 mais du monde fictionnel 1 construit et reconstruit au monde fictionnel 2 construit et reconstruit 2 (voir figure page suivante).

La reconstruction implique une interaction entre le lecteur et le texte selon des modèles cognitifs incluant des informations d'ordre linguistique, culturel, esthétique et littéraire de nature intertextuelle et transtextuelle. La pragmatique littéraire est un domaine vaste sinon illimité, puisqu'elle peut concerner tout individu et tout groupe d'individus; tout individu puisque comme le souligne Searle (1995) "l'ontologie réelle des états mentaux est une ontologie à la première personne ». La pragmatique du mentalais est un champ sans bornes.

Le poème de W. H. Auden "The Three Companions 》, tiré du recueil The Orators (1932), est le genre de poème-cryptogramme qui désespère traducteur et herméneute. Ce poème énigmatique est construit comme un dialogue avec trois paires de figures allégoriques et quasi paronomastiques : "reader to rider ", "fearer to farer ", "horror to hearer " avec inversion des paires dans le dernier quatrain. La structure allégorique ternaire éveille des échos intertextuels de dialogues entre vivants et morts : le thème des trois morts et des trois vifs chez Beaudoin de Condé, chez Nicolas de Margival, et qu'on retrouve en Allemagne, en Angleterre et en Italie aux XIII ${ }^{\circ}$ et $\mathrm{XIV}^{\circ}$ siècles (ex. La leggenda de tre morti et dei tre vivi, Le dit des trois morts et des trois vifs). Une étude poussée pourrait sûrement révéler d'autres textes de même nature. Les

${ }^{6}$ Celui qui fait de la métaphrase, «travail particulier qui explique par une tournure plus simple ou plus habituelie la phrase figuree, elliptique ou trop difficile. » (H. Lausberg, 1960, p. 246) 
Schema 8

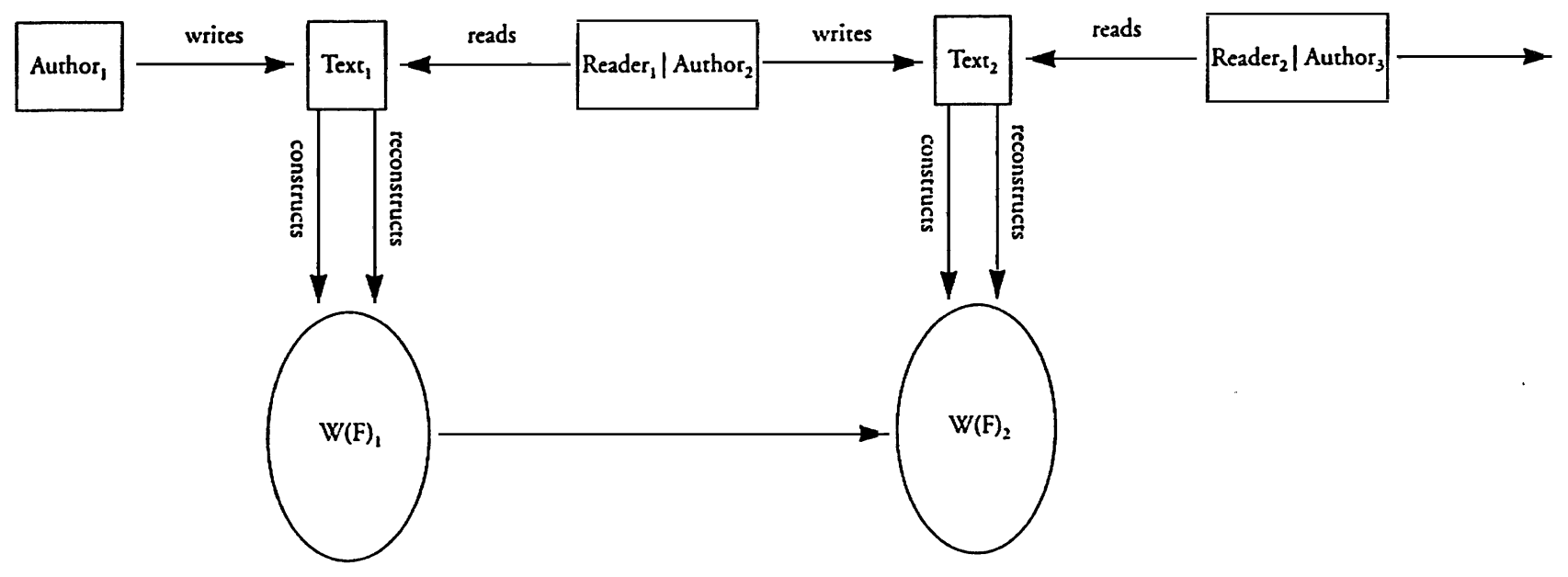


dictionnaires d'iconographie, d'emblématique et de devise illustrent à l'envi que les figures trinitaires constituent un lieu topique dont on pourrait tisser la transtextualité à travers les siècles. Vers la même époque, un peu antérieurement au poème "The Three Companions", nous découvrons chez W. B. Yeats les poèmes "The Three Hermits" et «The Three Beggars ». Mon propos n'est pas d'analyser ici cette tradition transallégorique dans le domaine littéraire mais de souligner certains traits inhérents au poème d'Auden. La référence y est presque absente, le temps et les lieux y sont rares, la sémantique intensionnelle, elle, est omniprésente. L'identité des personnages, celle du narrateur sont secrètes. Dans ce poème-cryptogramme l'allégorie est une métaphore continue qui nous donne un autre sens, caché, mais qui, ici, n'est pas explicité. L'allégorie opère dans le mystère - le lien de l'allégorie et du mysterium dans les littératures anciennes et médiévales - et l'atmosphère quelque peu méphitique comme dans les récits médiévaux et les allégories baroques où le cadavre est mis en représentation. Le réseau sémantique est ici plus allusif (horror, fatal, midden, madden, grave, dusk, twisted, skin, shocking disease).

Le traducteur, qui en tout état de cause s'efforce de garder l'esprit de la sémantique intensionnelle de l'original, et surtout des noms propres - par exemple le nom propre Méphisto est gardé tel quel dans les traductions -, ne peut le faire dans le cas de ce poème. C'est une impossibilité puisque les impersonnifications des paires allégoriques fearer/farer et les autres constituent de véritables paronomases dans le système de la langue anglaise, paronomases qu'il faut tenter de traduire dans un autre système de signes. Il y a dans la reconstruction par le lecteur ou le traducteur une allégorèse de fait. Ainsi, certaines traductions proposent pour reader/rider : lecteur/coureur ou liseur/rôdeur; pour fearer/farer : peureux/curieux ou peur/voyageur; pour horror/hearer : horreur/entendeur ou horreur/oreille.

La poésie contemporaine fait songer par le jeu de ses modalités à " la tonalité flottante» (schwebende Tonalität) de la musique dodécaphonique. De manière analogique je forme l'expression modalité flottante, inspirée de plusieurs sources : de la logique de Leibniz, de la logique moderne et plus étonnamment des écrits théoriques d'Anton Webern qui en 1933 a écrit un ouvrage Der Weg zur neuen Musik (La voie vers la musique moderne), titre donné en fait par Arnold Schönberg. Remarquons qu'Auden écrit "The Three Companions" à peu près au moment où Webern utilise l'expression schwebende Tonalität, tonalité flottante, pour expliquer l'évolution radicale de la musique occidentale dont il énumère les différentes phases:musique grégorienne et ses différents tons, développement de la musique polyphonique, construction de la ligne mélodique majeur et mineur, développement des tons bien tempérés dans la musique classique et romantique et enfin éclatement de la tonalité (Sprengung der Tonalität) dans ce qu'on nomme musique sérielle, dodécaphonique et parfois atonale. Il s'agit ici de la conquête systématique des touches noires et de la gamme chromatique. Pour faire 
bref, je dirai que dans le principe de base de cette création musicale inventée par Arnold Schönberg on utilise les douze tons de la gamme chromatique sans la moindre répétition des notes (pour ne pas évoquer et retrouver une tonalité quelconque, par exemple ré majeur, sol mineur, etc., tonalités auxquelles nos oreilles sont habituées depuis fort longtemps). Anton Webern fait remarquer que cette façon de composer rend la compréhension (die Fasslichkeit) beaucoup plus difficile. On pourrait développer plus longuement l'analogie musique/poésie entre la musique dodécaphonique et la poésie moderne (nouvelle version du $u t$ musica poesis) de cette même époque. On peut établir une série d'équations analogiques qui ne me semblent pas arbitraires pour la compréhension de certaines cuvres poétiques particulièrement énigmatiques ou de certaines œuvres dodécaphoniques. On remarquera d'abord souvent l'extrême brièveté de certains poèmes et de certaines compositions musicales, ce qui entraîne un certain nombre de conséquences, tant pour la forme que pour le sens et pour la condensation des effets utilisés. La troisième pièce de l'opus 11 d'Anton Webern se résume à dix courtes mesures; la quatrième de l'opus 10 dure une vingtaine de secondes. Certains poèmes modernes ne sont constitués que de quelques vers, si ce n'est même que de quelques mots. Dans les cuvres dodécaphoniques la tonalité est flottante et même éclatée et la référence par rapport aux tonalités antérieures est quasi impossible, d'où la difficulté pour de très nombreux auditeurs de saisir ou comprendre cette musique. Cependant la compréhension (Fasslichkeit) est possible, selon Webern, par une condensation de certains procédés formels : la série, la répétition, le renversement (Umkehrung), le retour (Krebs). Webern a une prédilection pour la forme courte, mais concentrée à un si haut degré qu'elle ne peut supporter un long développement.

En résumé on avancera sans trop forcer le trait que, dans la poésie contemporaine, les modalités flottantes s'expriment à différents niveaux :

1) d'un point de vue générique par le métissage des formes et des genres, par l'éclatement et la recomposition des éléments, par la diversité des supports et du médium graphique, plastique, visuel, audio-visuel et par leur métissage;

2) par l'indécidabilité des frontières entre monde actuel, mondes possibles, monde fictionnel et monde virtuel, par le fait que les mondes possibles et impossibles sont, en termes leibniziens, compossibles;

3) par la difficulté, qui existe parfois à évaluer la nature et le degré ontologique de tel poème, par le jeu souvent indécis entre les modalités aléthiques, déontiques et axiologiques diverses, et en définitive par la nature profondément subjective et individuelle de notre mentalais et par l'étendue, mal établie, de notre ignorance;

4) par la difficulté à saisir les processus de construction et de 
reconstruction du poème et par l'étendue des recherches pragmatiques envisageables pour la lecture ou la traduction.

Le poème-cryptogramme trouve son expression la plus étrange, la plus déroutante et la plus extrême chez les glossolales et les lettristes, dans le rythme inouï de l'énonciation et de la création de formes, de mots nouveaux, sauvages, plus ou moins interprétables ou compréhensibles, de jargons élucubrés/élucubrants par rapport aux modèles des langues véhiculaires ou vernaculaires. La glossolalie (glossa, glotta, parole et lalein/parler), le phénomène de lallation (dire la la la ou lambdacisme) se situent à la source articulatoire de la langue (langue, écriture comme souvenir de la tétée, écriture qui se constitue comme lallation, gazouillis, babillage). Les poètes lettristes et leur invention délirante d'une écriture traduisent une pulsion de l'inexprimable par un dicible au-delà des mots, par le jeu des lettres, des syllabes et des sonorités. On rappellera que la glossolalie est avant tout une langue inintelligible, propre aux mystiques au début de l'extase dans un contexte social, que la glossolalie est aussi un don surnaturel, jamais véritablement vérifié par les linguistes, de parler spontanément une langue étrangère, que la glossolalie est le langage imaginaire des aliénés, des illuminés, de certains mystiques et de certains écrivains. L'écrivain est le Protée capable de toutes les régressions et de toutes les projections futures; il peut en cela désespérer le traducteur. Autre paradoxe de la glossolalie, cette écriture qui est d'une singularité absolue vise en quelque sorte à être une langue universelle, une pasigraphie (du grec pas $=$ tout), une écriture pouvant être entendue de tous, partout, par l'utilisation de lettres, de signes, d'idéogrammes, de pictogrammes, de chiffres, de chiffres inventés. Certaines écritures de la poésie contemporaine révèlent une intention pasigraphique. La pulsion glossalique révèle un amour de la langue, par la relance systématique de la création morphophonologique et sémantique, par la répétition, par l'engendrement pulsionnel phonétique, lexical et morphosyntaxique. Dans le domaine artistique et ontologique on peut parler de loquacité excessive, de lalomanie, de logorrhée chez certains orateurs ou écrivains, de lalopathie et même, d'un point de vue psychiatrique, de lalopathologie. La fantasmagoria est une des manifestations profondes de la poésie.

La poétique est-elle ars conjectandi, camera obscura, art de mettre en cuvre des éléments et systèmes virtuels? Les recherches futures de notre millénaire seront-elles celles, toujours, de l'infiniment grand, de l'infiniment petit et... de l'infiniment complexe de notre langage et paysage mental, dans le monde, et de ses relations avec le système cortical, limbique, sympathique et parasympathique?

Université Carleton 


\section{Références}

BEN-PORAT, Ziva (1996). "Cognitive Poetics and experimental Study of Literature in Literary Pragmatics ", Language and Literature Today. Proceedings of the XIX'th Congress of the International Federation for Modern Languages and Literatures, Brasilia, 22-30 août 1993. Universidade de Brasilia, vol. 2, pp. 818-829.

CARAVETTA, Peter (1991). Prefaces to the Diaphora, Rhetorics, Allegory, and the Interpretation of Postmodernity. Purdue University Press.

DOLEŽEL, Lubomir (1998). Heterocosmica: Fiction and Possible Worlds. Baltimore, Johns Hopkins University Press.

-(1990). Occidental Poetics : Tradition and Progress. Lincoln, University of Nebraska Press.

FONTENELLE, Bernard (Le Bovier de) (1991). Euvres Complètes. Paris, Fayard.

HAGÈGE, Claude (1994). «Linguitique : pragmatisme et conceptualisme ", Raison présente. № 109.

HARTLIEB VON WALLTHOR, Alfred et QUIRIN, Heinz, éds. (1975). "Landschaft" als interdisziplinäres Forschungsproblem. Vorträge und Diskussionen des Kolloquiums am 7-8. November 1975 in Münster. Münster, Aschendorff.

HEIDEGGER, Martin (1982). "Hölderlins Hymne "Andenken" ", Gesammtausgabe. Frankfurt am Main, Vittorio Klostermann, Band 52.

- (1984). « Hölderlins Hymne "Der Ister" ». Frankfurt am Main, Vittorio Klostermann, Band 53.

HOPKINS, Gerard Manley (1959). Journals and Papers. London, Oxford University Press.

INGARDEN, Roman (1968). Vom Erkennen des literarischen Kunstwerks. Tübingen, Niemeyer.

KUBCAK, Hartmut (1975). Das Verhältnis von Intension und Extension als sprachwissenschaftliches Problem. Tübingen, Gunter Narr.

FODOR, Jerry A. (1994). The Elm and the Expert. Mentalese and its Semantics. Cambridge, Mass. MIT Press. 
FODOR, Jerry A. et LEPORE, E. (1992). Holism. A Shopper's Guide. Oxford, Blackwell.

LAURETTE, Pierre (1996). "Vers une grammaire de l'esprit postmoderne. Postmodernisme et métalangage ", N. de Faria (ed.), Language and Literature Today. Proceedings of the XLXth Congress of the International Federation for Modern Languages and Literatures, Brasilia, 22-30 août 1993. Universidade de Brasilia, pp. 15-27.

- (1993). Lettres et Technè. Informatique, instrumentations, méthodes et théories dans le domaine littéraire. Montréal, Balzac, coll. "L'Univers des discours" .

LAURETTE, Pierre et RUPRECHT, Hans George, éds. (1995). Poétiques et imaginaires. Francopolyphonie littéraire des Amériques. Paris, L'Harmattan.

LAUSBERG, Heinrich (1960). Handbuch der literarischen Rhetorik. Munich, Max Hüber Verlag.

MALLARMÉ, Stéphane (1945). "Crise de Vers », CEuvres complètes. Paris, Gallimard.

NOVALIS (1946). Gesammelte Werke. vol. 3, Zürich, Bühl Verlag.

PAULHAN, Jean (1966). CEuvres complètes. t. 2, Paris, Gallimard.

PAVEL, Thomas (1988). Univers de la fiction. Paris, Seuil.

PINKER, Steven (1997). How the Mind Works. New York/London, Norton.

- (1994). The Language Instinct. New York, William Morrow.

PORTEVUS, J. Douglas (1990) Landscape of the Mind, Worlds of Sense and Metaphor. Toronto, University of Toronto Press.

ROGER-MARX, Claude (1952). Le paysage français. De Corot à nos jours ou le dialogue de l'homme et du ciel. Paris, Plon.

SEARLE, John (1995). La redécouverte de l'esprit. Paris, Gallimard.

SPERBER, Dan (1992). " Les sciences cognitives, les sciences sociales et le matérialisme ", dans Daniel Andler (éd.), Introduction aux sciences cognitives. Paris, Gallimard.

RÉSUMÉ : L'hologramme du poème et le prisme du poétique. Paysages. Mondes possibles. Langage de la pensée - Une relecture de la rhétorique-poétique est proposée à la lumière des développements 
récents de la théorie littéraire et de certains aspects des sciences cognitives. La poésie est abordée plus précisément sous le rapport des mondes possibles ainsi que de la traduction-transduction, théorie qui réintègre la sémantique intensionnelle et extensionnelle dans l'analyse littéraire. Toutefois, la poésie moderne ne se laisse pas réduire à cette analyse philosophico-logique. La complexité du langage poétique oblige à envisager aussi la poésie du point de vue de la pensée et du langage (le mentalais) axé non seulement sur le cortical et le cérébral mais également sur l'intuition et les sensations (le limbique).

ABSTRACT : The Hologram of Poetry and the Prism of Poetics. Landscapes. Possible Worlds. The Language of Thought - A rereading of poetics-rhetorics is proposed here, in light of recent developments in literary theory and of certain aspects of cognitive science. Poetry is approached more precisely in the relationship between possible worlds, as well as translation-transduction, a theory reintegrating intensional and extensional semantics in literary analysis. However, modern poetry cannot be reduced to this philosophico-logical analysis. The complexity of poetic language obliges poetry to be envisioned from the point of view of thought and language (mentalese), articulated not only on the cortical and cerebral, but on intuition and sensations as well (the limbic).

Pierre Laurette : Department of French, Dunton Tower, Carleton University, 1125 Colonel By Drive, Ottawa (Ontario) K1S 5B6 